\title{
Mechanism of central temperature peaking in impurity seeded plasmas
}

\author{
M Z Tokar \\ Institut für Plasmaphysik, Forschungszentrum Jülich GmbH, Association FZJ-Euratom, 52425,
} Jülich, Germany

Received 12 February 2003, in final form 29 April 2003

Published 24 June 2003

Online at stacks.iop.org/PPCF/45/1323

\begin{abstract}
Formation of a centrally peaked profile of the ion temperature by impurity seeding has been observed in all the largest tokamaks: JET, JT-60U, TFTR. A possible explanation of this phenomenon is proposed, which takes into account the suppression of ion temperature gradient instability induced anomalous transport through increasing ion effective charge $Z_{\text {eff }}$ and Doppler shift of unstable modes, $S_{\perp}$. The particular importance of the $S_{\perp}$ component proportional to the temperature gradient, which stems from the pressure gradient and neoclassical poloidal rotation, is elucidated. It is demonstrated that this contribution grows significantly by impurity seeding when the rotation regime changes due to increased collisionality. An interrelation between the heating power density and $Z_{\text {eff }}$ necessary for the transition to a peaked ion temperature is found. The modification of radial profiles by impurity seeding is modelled numerically and the results are in agreement with the experimental data from JET.
\end{abstract}

\section{Introduction}

Experiments with deliberate seeding of impurities were performed on several fusion devices including tokamaks and stellarators [1]. The initial goal of these studies was to reduce the concentration of energy losses onto the wall elements exposed to intensive heat loads, i.e. limiters and divertor plates, by increasing the edge radiation [2]. Astonishingly, these experiments have revealed another beneficial effect from impurities, namely, a significant reduction of the anomalous transport of energy and particles in hot plasmas. Results of transport modelling demonstrate [3-5] that this occurs owing to the suppression of the ion temperature gradient (ITG) instability, which dominates the anomalous losses in standard situations. Depending on experimental conditions different manifestations of reduced transport and improved confinement have been observed.

In devices of moderate dimensions with a relatively low plasma temperature, a significant peaking of the electron density $n_{e}$ was recorded $[6,7]$, which plays an important role in the suppression of ITG turbulence [3,4]. In [8], the transition to the state with peaked $n_{e}$ 
and improved confinement was explained as a bifurcation in the radial electron flux, $\Gamma_{e}$, generated by ITG and dissipative trapped electron (DTE) unstable modes. According to [9] the contribution of ion driven turbulence to $\Gamma_{e}$ is predominantly of a diffusive nature. In contrast, the DTE transport causes a much stronger inward particle convection proportional to the magnetic shear. An increase of $Z_{\text {eff }}$ with impurity seeding gives an initial 'kick' to the reduction in ITG growth rate and particle diffusion. Due to an inward pinch induced by DTE-modes the density peaks. This suppresses ITG transport further and if $Z_{\text {eff }}$ is high enough a bifurcation to radiative improved (RI) mode can occur [8].

In the case of unbalanced neutral beam injection (NBI) applied for plasma heating, a fast toroidal plasma rotation is normally induced. Its velocity $V_{\varphi}$ is essentially controlled by the perpendicular plasma viscosity. ITG turbulence provides the main contribution to this transport channel $[10,11]$. The suppression of ITG unstable modes with rising $Z_{\text {eff }}$ results in a steeper radial profile of $V_{\varphi}$ and, through the ion force balance [12], in a sharper gradient of the radial electric field $E_{r}$. The Doppler shift $S_{\perp}$ of perturbations due to the drift motion induced by $E_{r}$ increases and kills turbulent eddies. This leads to reduced anomalous transport [12] and can cause a transition to a state with completely suppressed ITG transport and a very sharp radial gradient of plasma rotation [13].

Another kind of phenomenon has been observed by impurity seeding into the largest tokamaks JET, JT-60U and TFTR [14-16]. In these devices, the most prominent manifestation of confinement improvement in the presence of impurities is a significant peaking of the ion temperature $T_{i}$ in the central plasma region, $r \leqslant r_{*} \ll a$, where $a$ is the minor radius $r$ of the last closed magnetic surface (separatrix). Figure 1 taken from [14] shows an example of such behaviour in JET L-mode plasmas seeded by neon. In this paper, we propose a model for this phenomenon, which allows us to find the conditions necessary for the formation of such a strongly peaked ion temperature profile in the plasma core. In addition, the radial profiles of $T_{i}$ and electron density are modelled numerically and the importance of density peaking for the evolution of the ion temperature is examined.

\section{Transport model}

In plasmas without seeded impurities when the stabilizing effect of the radial electric field on ITG turbulence is weak, the contribution of this transport channel to the ion heat conductivity $\chi_{i}$ can be estimated in mixing length approximation [18]:

$$
\chi_{\mathrm{ITG}} \approx \xi \frac{\gamma_{\max }}{k_{\max }^{2}}
$$

Here, $k_{\max }$ is the wave number of the most unstable perturbations and in a deuterium plasma $k_{\max }^{2} \rho_{s}^{2} \approx 0.2$ (see, e.g. [4]) with

$$
\rho_{s}=\frac{c_{s}}{\omega_{\mathrm{ic}}}, \quad c_{s}=\sqrt{\frac{T_{e}}{m_{i}}}
$$

and $\omega_{\mathrm{ic}}$ being the Larmor radius, sound velocity and cyclotron frequency, respectively, of the background ions. The maximum growth rate $\gamma_{\max }$ of ITG-modes can be estimated according to the formula [8]

$$
\gamma_{\max } \approx \frac{c_{s}}{\sqrt{\alpha Z_{\mathrm{eff}} R}} \sqrt{\frac{1}{L_{T_{i}}}-\frac{1}{L_{T_{i}}^{\mathrm{cr}}}}
$$




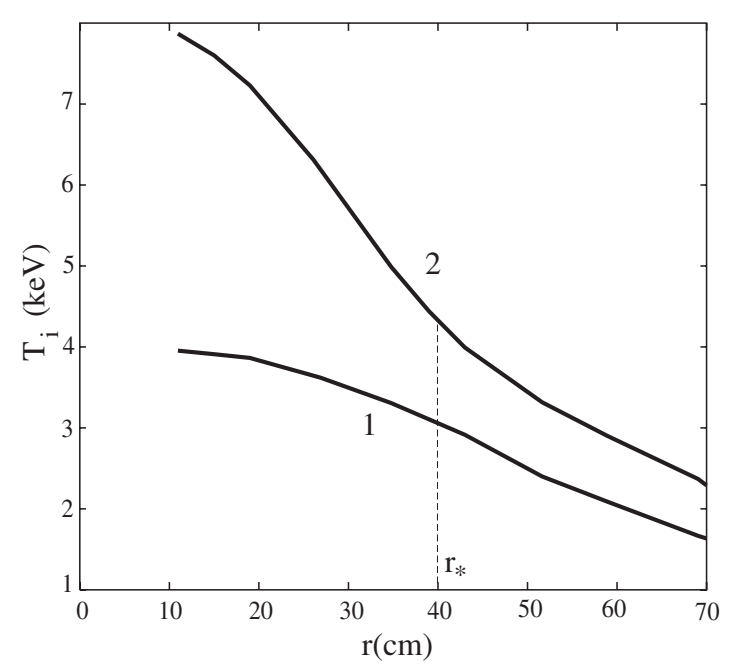

Figure 1. The experimental profiles from [14] of the ion temperature in JET L-mode reference shot 53156 (curve 1) and in shot 53154 under conditions of intense neon seeding (curve 2) obtained at $44.8 \mathrm{~s}$; the profiles measured by charge-exchange recombination spectroscopy of carbon ions normally have an error of $15 \%$ [17]; $r_{*}$ is the radius of the magnetic surface inside which significant difference in the temperature gradient can be seen, the separatrix radius $a=90 \mathrm{~cm}$.

where $R$ is the torus major radius, $L_{T_{i}} \equiv-\mathrm{d} \ln T_{i} / \mathrm{d} r$ the ion temperature $e$-folding length; its critical value, at which $\gamma_{\max }$ reduces to zero, is given by the relation:

$$
\frac{1}{L_{T_{i}}^{\mathrm{cr}}}=\frac{4}{3 R}\left(1+\frac{1}{\alpha Z_{\mathrm{eff}}}\right)+\frac{2 \alpha Z_{\mathrm{eff}}}{R}\left(\frac{2}{3 \alpha Z_{\mathrm{eff}}}-\frac{1}{2}-\frac{R}{4} \frac{\mathrm{d} \ln n_{e}}{\mathrm{~d} r}\right)^{2}
$$

being a generalization of the one in [18] on the case of arbitrary $Z_{\mathrm{eff}}$. Here, $\alpha=T_{e} / T_{i}$ and by taking as a basis the experimental data from similar JET shot 50329 (see [19]) we assume $\alpha \approx 0.6$ independently of $r$. The numerical factor $\xi \approx 0.7$ was introduced in order to reproduce the experimental value of an ion heat conductivity of $2-3 \mathrm{~m}^{2} \mathrm{~s}^{-1}$ from the TRANSP modelling in the case without seeded impurities [14].

From equation (2), one can see that impurities have a direct effect on ITG induced anomalous transport. This is explained by the fact that toroidal ITG instability develops because the diamagnetic drift is not divergence free in the tokamak magnetic geometry. The drift velocity is inversely proportional to the ion charge and therefore $\chi_{\text {ITG }}$ drops with growing impurity concentration. However, when the impurity charge contribution becomes more than about $50 \%$ of the electron charge the impurity ITG-mode becomes dominant over the hydrogenic ITG-mode [20]. Equations (1)-(3) take this transition into account and describe the saturation of $Z_{\text {eff }}$ effect on $\chi_{\mathrm{ITG}}$. The total reduction of $\chi_{\mathrm{ITG}}$ when $Z_{\text {eff }}$ grows from 2 to 6 is of $30-35 \%$.

In addition to this effect there is an indirect influence of impurities on ITG turbulence coming into play through the stabilizing role of the radial electric field. In the presence of the radial electric field $\gamma_{\max }$ is reduced by the Doppler shift [21]:

$$
S_{\perp}=\left|\frac{\mathrm{d} V_{i \varphi}}{\mathrm{d} r} \frac{B_{\vartheta}}{B}-r \frac{\mathrm{d}}{\mathrm{d} r}\left(\frac{V_{i \vartheta}}{r}\right)+\frac{c r}{e B} \frac{\mathrm{d}}{\mathrm{d} r}\left(\frac{1}{r n_{i}} \frac{\mathrm{d} n_{i} T_{i}}{\mathrm{~d} r}\right)\right|
$$

Here, $B_{\vartheta}$ is the poloidal component of the magnetic field, $V_{i \varphi}$ and $V_{i \vartheta}$ are the toroidal and poloidal components of the ion velocity. Both $B_{\vartheta}$ and the derivative of $V_{\varphi}$ reduce to zero at 
the plasma axis and therefore we neglect the first term in $S_{\perp}$ in the central region in question. Indeed, implying a parabolic profile for $V_{i \varphi}, V_{0}\left(1-r^{2} / a^{2}\right)$, and using the definition of the safety factor $q$, we get

$$
\left|\frac{\mathrm{d} V_{i \varphi}}{\mathrm{d} r} \frac{B_{\vartheta}}{B}\right| \approx \frac{2 V_{0}}{q R} \frac{r^{2}}{a^{2}}
$$

For $q \approx 1, R=3 \mathrm{~m}$ and a measured $V_{0}$ of $125 \mathrm{~km} \mathrm{~s}^{-1}$ [14], this gives a value of $4000 \mathrm{~s}^{-1}$ at $r=25 \mathrm{~cm}$ where the difference in the temperature gradients is the most pronounced. This is by an order of magnitude smaller than a contribution of $40000 \mathrm{~s}^{-1}$ from the pressure term estimated for $B_{T}=2.2 \mathrm{~T}$ [14] by using the temperature profile given by curve 2 in figure 1 . Moreover, the density profile is much flatter in the plasma core than that of the temperature. Therefore, the radial variation of $n_{i}$ can be neglected in the last pressure driven term. In addition, we assume neoclassical approximation for the poloidal component of the ion velocity [22]:

$$
V_{i \vartheta}=k_{\text {neo }}^{i} \frac{c}{e B} \frac{\mathrm{d} T_{i}}{\mathrm{~d} r}
$$

Finally, one gets

$$
S_{\perp} \approx \frac{c r}{e B}\left|\frac{\mathrm{d}}{\mathrm{d} r}\left(\frac{1-k_{\text {neo }}^{i}}{r} \frac{\mathrm{d} T_{i}}{\mathrm{~d} r}\right)\right|
$$

The neoclassical approximation can be questioned close to the plasma axis where $B_{\vartheta}$ reduces to zero. A firm theory for the plasma rotation in this case does not exist yet, but one can expect a significant deviation from the standard neoclassics for radii smaller than the width of 'potato' orbits: $\Delta r_{p} \sim(2 q \rho)^{2 / 3} R^{1 / 3}$ [23]. Under the conditions in question the ion poloidal velocity is essentially determined by impurities and namely their larmor radius $\rho$ should be used by estimating $\Delta r_{p}$. For JET parameters under consideration this results in $\Delta r_{p} \leqslant 3 \mathrm{~cm}$, i.e. much narrower than the region where the temperature peaking is localized, $r \leqslant r_{*} \approx 30-40 \mathrm{~cm}$.

The factor $k_{\text {neo }}$ changes significantly between different regimes of neoclassical transport characterized by the ion collisionality $\nu_{*} \equiv\left(q R / \lambda_{i}\right) \varepsilon^{-3 / 2}$, with $\lambda_{i}$ being the mean free path length of ions between coulomb collisions and $\varepsilon=r / R$. In the banana regime, where $v_{*} \ll 0.302$ [24], $k_{\text {neo }} \approx 1.17$; in the plateau with $0.302 \ll v_{*} \ll 0.222 \varepsilon^{-3 / 2}[24], k_{\text {neo }} \approx-0.5$ and $k_{\text {neo }} \approx-1.83$ in the Pfirsch-Schlüter regime where $0.222 \varepsilon^{-3 / 2} \ll v_{*}$ [24]. These limit values are interpolated by the formula [25]:

$$
k_{\mathrm{neo}} \approx-1.83 \frac{v_{*} \varepsilon^{3 / 2}+0.061}{v_{*} \varepsilon^{3 / 2}+0.222} \frac{v_{*}-0.703}{v_{*}+0.302}
$$

Normally at the plasma axis where $\varepsilon, r=0$ the ion temperature is high enough so that $\lambda_{i} \sim T_{i}^{2}$ significantly exceeds $q R$ and the background ions are in the plateau regime. With increasing $r$ the transition to the banana regime takes place and both $\left|1-k_{\text {neo }}\right|$ and $S_{\perp}$ decrease by an order of magnitude so that the stabilizing effect of Doppler shift reduces drastically. By impurity seeding the mean free path length of ions decreases as $1 / Z_{\text {eff }}$ and the plateau region, where the stabilization effect is strong, expands. In addition, coulomb collisions between main and impurity ions bring their velocities closer to each other. The effective ion poloidal velocity $V_{\vartheta}$ is determined by the momentum conservation:

$$
V_{\vartheta}\left(n_{i} m_{i}+n_{Z} m_{Z}\right)=V_{i \vartheta} n_{i} m_{i}+V_{Z \vartheta} n_{Z} m_{Z}
$$

Here $n_{Z}$ and $m_{Z}$ are the density and mass of the dominant impurity ions of the charge $Z$ and their neoclassical velocity

$$
V_{Z \vartheta}=k_{\text {neo }}^{Z} \frac{c}{Z e B} \frac{\mathrm{d} T_{i}}{\mathrm{~d} r}
$$


with $k_{\text {neo }}^{Z}$ given by equation (6) where $v_{*}$ is calculated with the mean free path of impurity ions. For a single impurity species one can relate the ion densities to that of the electron one:

$$
n_{i}=n_{e} \frac{Z-Z_{\mathrm{eff}}}{Z-1} \quad \text { and } \quad n_{Z}=\frac{n_{e}}{Z} \frac{Z_{\mathrm{eff}}-1}{Z-1}
$$

For the effective $k_{\text {neo }}$, which should be used in equation (5) instead of $k_{\text {neo }}^{i}$ in the case of an impure plasma, we get

$$
k_{\text {neo }}=\frac{k_{\text {neo }}^{i}+k_{\text {neo }}^{Z}(\varsigma / Z)}{1+\varsigma} \quad \text { with } \varsigma=\frac{Z_{\text {eff }}-1}{Z-Z_{\text {eff }}} \frac{m_{Z}}{Z m_{i}}
$$

Earlier [26], the importance of the collisionality dependence of the neoclassical poloidal rotation in the $E \times B$ velocity shear was demonstrated for the formation of edge transport barriers.

When the ITG induced anomalous transport is suppressed the ion heat transfer is dominated by neoclassical losses. Following [14], we assume a value of $0.2 \mathrm{~m}^{2} \mathrm{~s}^{-1}$ for the corresponding contribution $\chi_{\text {neo }}$ to the ion heat diffusivity and $0.5 \mathrm{~m}^{2} \mathrm{~s}^{-1}$ - for the electron heat diffusivity $\chi_{e}$. Henceforth, it will be assumed that both $\chi_{\text {neo }}$ and $\chi_{e}$ do not change noticeably with the plasma conditions. Note, the estimate for $\chi_{\text {neo }}$ was obtained in [14] from the interpretive code TRANSP by using not the most sophisticated model of neoclassical transport. Inaccuracy of this estimate should not, however, be of much importance since the conclusions drawn in this paper do not change significantly by a variation of $\chi_{\text {neo }}$ in the wide range $0.1-0.4 \mathrm{~m}^{2} \mathrm{~s}^{-1}$.

\section{Bifurcation to centrally peaked temperature profile}

In this section, we consider analytically under which conditions ITG transport can be suppressed in the central plasma region $r \leqslant r_{*}$ and a strongly peaked temperature profile develops. The profile peakedness here is characterized by the value

$$
\theta=\frac{T_{i}(r=0)-T_{* i}}{T_{* i}} \quad \text { with } T_{* i}=T_{i}\left(r_{*}\right)
$$

assumed henceforth as a given. Consider the plasma heat balance inside the magnetic surface where the temperature gradient approaches its maximum, i.e. roughly at $r=0.5 r_{*}$ :

$$
\pi\left(\frac{r_{*}}{2}\right)^{2} Q_{\text {heat }}=-2 \pi \frac{r_{*}}{2}\left[\left(n_{i}+n_{Z}\right) \chi_{i} \frac{\mathrm{d} T_{i}}{\mathrm{~d} r}+n_{e} \chi_{e} \frac{\mathrm{d} T_{e}}{\mathrm{~d} r}\right]
$$

with the heating density from NBI, $Q_{\text {heat }}$, adopted independent of $r$. By using the estimates

$$
\frac{\mathrm{d} T_{i}}{\mathrm{~d} r} \approx \frac{\theta T_{* i}}{r_{*}} \quad \text { and } \quad L_{T_{i}} \approx r_{*} \frac{2+\theta}{2 \theta}
$$

we obtain from this relation an equation for $\theta$ :

$Q_{\text {heat }}=\frac{4 T_{* i} n_{* e}}{r_{*}^{2}}\left[\left(1-\frac{Z_{\text {eff }}-1}{Z}\right)\left(\chi_{L} \sqrt{\frac{\theta}{2+\theta}-\frac{r_{*}}{2 L_{T_{i}}^{\mathrm{cr}}}}-\chi_{S} \theta+\chi_{\text {neo }}\right)+\alpha \chi_{e}\right] \theta \equiv P(\theta)$

where

$$
\chi_{L}=5 \sqrt{2} \frac{\xi c_{s} \rho_{s}^{2}}{\sqrt{R r_{*} Z_{\mathrm{eff}}}}, \quad \chi_{S}=\frac{10}{\alpha} \frac{c_{s} \rho_{s}^{3}}{r_{*}^{2}}\left|1-k_{\mathrm{neo}}\right|
$$

and $k_{\text {neo }}^{i}$ was assumed constant in the region in question.

Figure 2 shows the function $P(\theta)$ for deuterium plasma in JET in shots without and with seeding of neon [14] computed with $r_{*}=0.4 \mathrm{~m}$. In the former case $n_{* e}=1.7 \times 10^{19} \mathrm{~m}^{-3}$, 


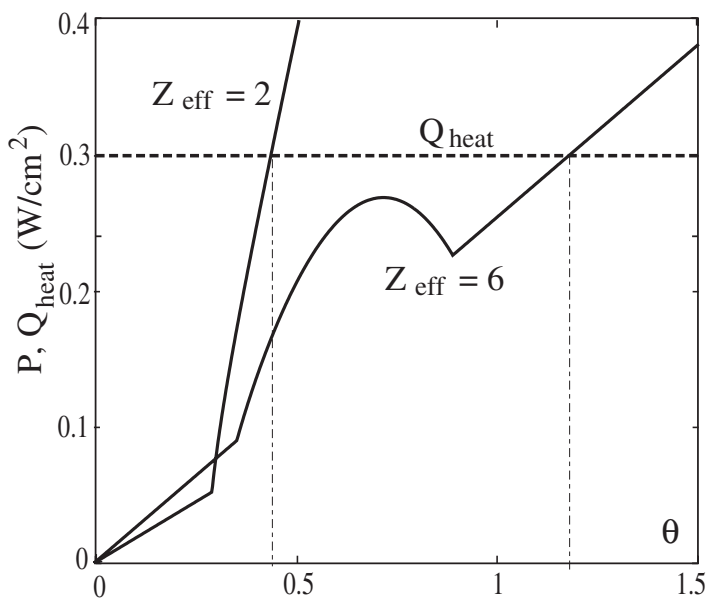

Figure 2. Function $P(\theta)$ and stationary states of the heat balance in the plasma core for different values of the effective ion charge.

$T_{* i}=3 \mathrm{keV}, Z_{\text {eff }}=2$ and $n_{* e}=2 \times 10^{19} \mathrm{~m}^{-3}, T_{* i}=4 \mathrm{keV}, Z_{\text {eff }}=6$ in the latter one. One can see that the function $P(\theta)$ can have a maximum: $\chi_{\text {ITG }}$ increases with $\theta$ as $\sqrt{\theta /(2+\theta)-r_{*} / 2 L_{T_{i}}^{\text {cr }}}$ but the reduction of anomalous transport by the Doppler shift grows linearly. In the first case $P_{\text {max }}$ is much larger than $Q_{\text {heat }} \approx 0.3 \mathrm{~W} \mathrm{~cm}^{-3}[14]$ and is achieved at very large $\theta$; the stationary $\theta$ value is small and the temperature profile in the plasma core is relatively flat. With increasing $Z_{\text {eff }} \chi_{L}$ diminishes and $\chi_{S}$ becomes larger since the plasma is more collisional and $k_{\text {neo }}$ tends to more negative values. Therefore, $P_{\max }$ decreases and the transition to a state with suppressed ITG turbulence occurs when $P_{\max }$ reduces to the $Q_{\text {heat }}$ level. In this state, the heat is lost mainly through the electron channel.

By taking into account that (i) $\theta_{\max }$, at which $P(\theta)$ approaches its maximum, exceeds noticeably $r_{*} / L_{T_{i}}^{\mathrm{cr}}$ and (ii) $\chi_{\mathrm{ITG}}\left(\theta_{\max }\right) \gg \chi_{\text {neo }}, \chi_{e}$, one can estimate $\theta_{\max }$ and $P_{\max }$ analytically:

$$
\begin{aligned}
& \theta_{\max } \approx \frac{9}{32}\left(\frac{\chi_{L}}{\chi_{S}}\right)^{2} \\
& P_{\max } \approx \frac{27}{256} \chi_{L} \frac{n_{* e} T_{* i}}{r_{*}^{2}}\left(\frac{\chi_{L}}{\chi_{S}}\right)^{3}\left(1-\frac{Z_{\mathrm{eff}}-1}{Z}\right)
\end{aligned}
$$

By making the substitutions for parameters $\chi_{L}$ and $\chi_{S}$ we get the following expression for the critical heating power density, at which the transition to the peaked temperature profile with large $\theta$ takes place:

$$
Q_{\text {heat }}^{\text {cr }} \approx 0.025 \frac{n_{* e} T_{* i} \omega_{\text {ic }}}{\left|1-k_{\text {neo }}\right|^{3}} \frac{r_{*}^{2}}{R^{2}} \frac{Z+1-Z_{\text {eff }}}{Z Z_{\text {eff }}^{2}}
$$

This formula predicts a reduction of $Q_{\mathrm{heat}}^{\mathrm{cr}}$ by an order of magnitude in JET when going from plasmas with intrinsic carbon impurity $\left(Z=6, Z_{\text {eff }}=2\right)$ to plasmas after heavy neon puffing $\left(Z=10, Z_{\text {eff }}=5\right.$ ). One can also see from equation (12) that the heating power density necessary for the transition increases with the minor radius of the central region where ITG transport is suppressed. This means that the reduction of transport occurs for all $r \leqslant r_{*}$ in agreement with the experimental observations $[14,15]$. 


\section{Results of numerical modelling}

In order to take into account the radial variation of $k_{\text {neo }}$, which is one of the key parameters in this model, the heat conduction equation:

$$
\frac{1}{r} \frac{\mathrm{d}}{\mathrm{d} r}\left(-r \kappa_{\mathrm{eff}} \frac{\mathrm{d} T_{i}}{\mathrm{~d} r}\right)=Q_{\text {heat }}
$$

with $\kappa_{\text {eff }}=\left(n_{i}+n_{Z}\right) \chi_{i}+\alpha n_{e} \chi_{e}$ has been integrated numerically with a value of $T_{i}$ at $r=r_{b}=60 \mathrm{~cm}$ assumed equal to the experimental one (see figure 1). This position of the computation domain boundary was chosen from the following point of view. According to the TRANSP results [14], by impurity seeding the most significant changes in the anomalous transport occur in the plasma core and at the edge. In this paper, we consider only the former effect of central temperature peaking which is localized in the region of $r \leqslant 40 \mathrm{~cm}$ (see figure 1). Thus, on the one hand, $r_{b}$ should be larger than this value. On the other hand, this model does not include important physical processes at the plasma edge, e.g. sources of charged particles, convective heat transport, radiation losses. Modelling by the code RITM [19] shows that these processes are of importance in the region of $r \geqslant 65 \mathrm{~cm}$, which sets the upper limit for $r_{b}$. By setting the experimental value for $T_{i}$ as the boundary condition at $r_{b}$ we implicitly take into account the modification of the edge confinement by impurity seeding. The result does not change strongly if $r_{b}$ varies in the range $40-65 \mathrm{~cm}$. The incorporation of this transport model into the code RITM will give a possibility of describing the impurity effect in the whole plasma self-consistently. Figure 3 shows the radial temperature profile calculated for different magnitudes of the ion effective charge. For $Z_{\text {eff }}=5$ the profile peaks in the central region with $r \leqslant 35 \mathrm{~cm}$ and its shape is in good agreement with experimental measurements (see figure 1).

Thus, the central peaking of the ion temperature, being the main cause of the global confinement improvement under conditions in question, can be explained by the effect of the radial electric field on ITG turbulence. This effect comes into play mainly through the contribution of the ion temperature gradient (ITG) to $E_{r}$. In smaller devices like TEXTOR the peaking of the electron density was identified as the main channel of improvement $[3,4,8]$. This mechanism can lead to suppression of ITG unstable modes since an increase of the density gradient above a certain level results in a reduction of the critical temperature $e$-folding length (see equation (3)). In JET and JT-60 experiments a moderate density peaking was observed and

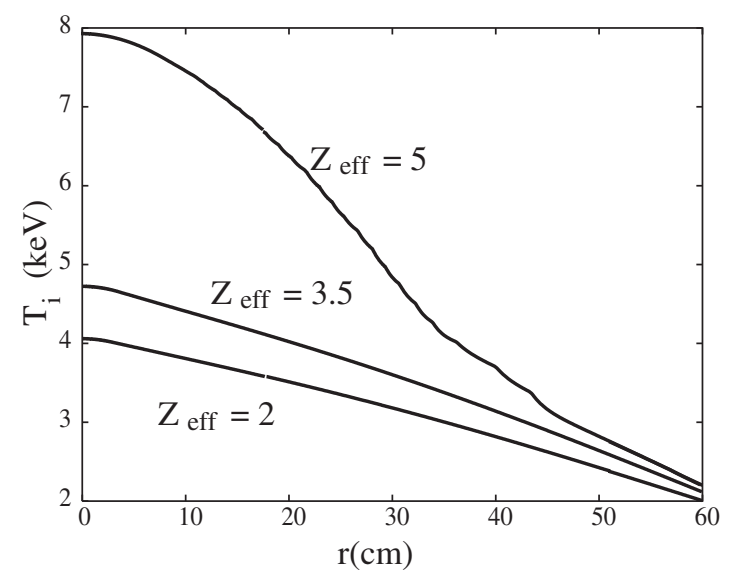

Figure 3. Radial profiles of the ion temperature computed for different $Z_{\text {eff }}$ by numerical integration of equation (13). 
it is of interest to analyse its importance for the central temperature evolution. For this purpose the density profile in the plasma core, $r \leqslant r_{b}$, was modelled by the continuity equation:

$$
\frac{1}{r} \frac{\mathrm{d}}{\mathrm{d} r}\left(r \Gamma_{e}\right)=\frac{Q_{\text {heat }}}{E_{\text {beam }}}
$$

where the RHS takes into account the plasma source due to NBI with $E_{\text {beam }}$ being the energy of beam particles.

The transport model described in $[8,19,27]$ was applied to compute the electron flux density,

$$
\Gamma_{e}=-D_{\perp} \frac{\mathrm{d} n_{e}}{\mathrm{~d} r}+V_{\perp} n_{e}
$$

with contributions to the particle transport coefficients from ITG and DTE unstable modes:

$$
\begin{aligned}
& D_{\perp}=\chi_{\mathrm{ITG}} f_{\mathrm{tr}}+\chi_{\mathrm{DTE}} \\
& V_{\perp}=-\left(\chi_{\mathrm{ITG}} f_{\mathrm{tr}} \frac{4 r}{3 R}+\chi_{\mathrm{DTE}}\right) \frac{\mathrm{d} \ln q}{\mathrm{~d} r}
\end{aligned}
$$

Here, $f_{\text {tr }} \approx \sqrt{2 r / R}$ is the fraction of trapped particles; the electron heat diffusivity due to DTE-modes, $\chi_{\mathrm{DTE}}$, is calculated in the mixing length approximation (see equation (1)) with the growth rate

$$
\gamma_{\max }=\frac{\mathrm{d} \ln T_{e}}{\mathrm{~d} \ln n_{e}} \frac{v_{\mathrm{eff}} \omega_{* e}^{2}}{\omega_{* e}^{2}+v_{\mathrm{eff}}^{2}} f_{\mathrm{tr}}
$$

[28] reduced by $S_{\perp}$, where

$$
v_{\mathrm{eff}}=\frac{R}{\tau_{e} r} \quad \text { and } \quad \omega_{* e}=\frac{c T_{e} k_{\max }}{e B}\left(-\frac{\mathrm{d} \ln n_{e}}{\mathrm{~d} r}\right)
$$

are the electron effective collision and drift frequencies, respectively, and $k_{\max }^{2} \rho_{s}^{2} \approx 1$.

Figure 4 demonstrates the density profiles computed for different magnitudes of the ion effective charge and reproduces well the density evolution observed by neon seeding into JET L-mode plasmas [19]. In order to analyse how far this modification of the density is of importance for the temperature peaking, equations (13) and (14) were solved under

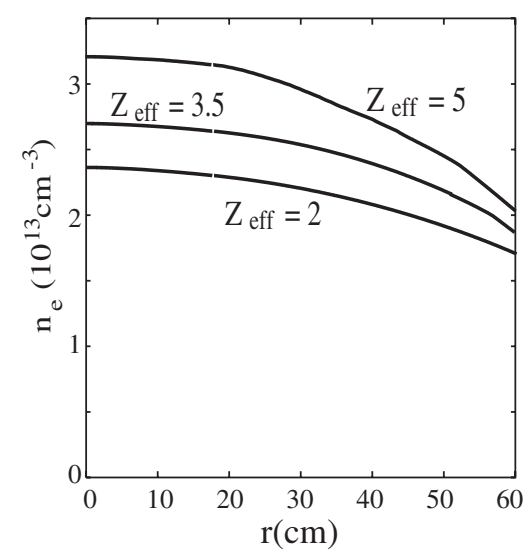

Figure 4. Radial profiles of the electron density computed for different $Z_{\text {eff }}$ by numerical integration of equation (14). 


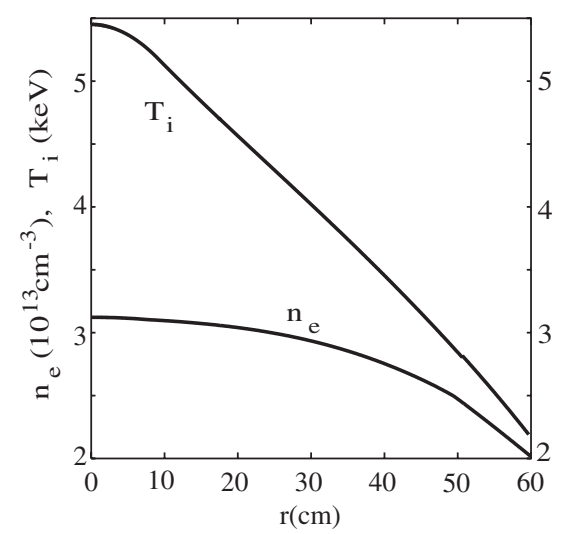

Figure 5. Radial profiles of plasma parameters calculated for $Z_{\mathrm{eff}}=6$ without taking into account ITG stabilization by the radial electric field.

assumption $S_{\perp}=0$. The ion temperature profile shown in figure 5 does not display any strong peaking even for $Z_{\text {eff }}=6$. Thus, the increase of $Z_{\text {eff }}$ and modification of the density profile alone cannot suppress ITG turbulence in the central plasma and the role of the radial electric field is decisive for temperature peaking. This situation differs principally from that at the plasma edge where ITG turbulence is suppressed predominantly by the density gradient and the region with reduced transport spreads by impurity seeding [19] as happens in the RI-mode in TEXTOR [7].

\section{Conclusion}

Formation of a centrally peaked ion temperature profile by seeding of impurities has been seen in all the largest tokamaks: JET, JT-60U and TFTR. The influence of the ion effective charge on anomalous transport due to ITG unstable modes through its direct effect on the linear growth rate and stabilization by the radial electric field provides a possible explanation for this phenomenon. In the latter case impurities come into play through the effect of $Z_{\text {eff }}$ on the neoclassical poloidal rotation. The contribution of this rotation to $E_{r}$ leads to much stronger stabilization in the near axis region where ions are in the plateau regime, than in the main plasma part where they are in the banana regime. By impurity seeding the plasma collisionality increases and the plasma region, where ions belong to the plateau regime, spreads out. The relation between the heating power density and $Z_{\text {eff }}$ required for bifurcation to a peaked temperature profile has been found from a zero-dimensional analytical analysis. Numerically computed radial profiles of the ion temperature and electron density are in agreement with experimental profiles from JET.

In conclusion, a very simple approximation for $V_{\vartheta}$ based on standard neoclassical theory has been used in this paper. This can be questionable close to the axis and in particular at vanishing $B_{\vartheta}$. Therefore, the results of such a reduced implementation should be checked in future by comparing them with predictions of more sophisticated approaches, e.g. from the code NCLASS [29].

\section{References}

[1] Ongena J et al 2001 Phys. Plasmas 82188

[2] Samm U et al 1993 Plasma Phys. Control. Fusion 35 B167 
[3] Sydora R et al 1996 Plasma Phys. Control. Fusion 38 A281

[4] Tokar M Z 1999 Plasma Phys. Control. Fusion 41 L9

[5] McKee G R et al 2000 Phys. Plasmas 71870

[6] Lazarus E A et al 1984 J. Nucl. Mater. 12161

[7] Weynants R R et al 1999 Nucl. Fusion 391637

[8] Tokar M Z et al 2000 Phys. Rev. Lett. 84895

[9] Baker D R and Rosenbluth M N 1998 Phys. Plasmas 52936

[10] Staebler G M and Dominguez R R 1993 Nucl. Fusion 3377

[11] Tokar' M Z et al 1998 Nucl. Fusion 38961

[12] Terry P W 2000 Rev. Mod. Phys. 72109

[13] Murakami M et al 2001 Nucl. Fusion 41317

[14] Jackson G L et al 2002 Plasma Phys. Control. Fusion 441893

[15] Hill K W et al 2002 Study of integrated high-performance regimes with impurity injection in JT-60U discharges 19th IAEA Fusion Energy Conf. (Lyon, France, 14-19 October 2002) p IAEA-CN-94/EX/P2-03

[16] Hill K W et al 2001 Phys. Plasmas 6877

[17] Boileau A et al 1989 Plasma Phys. Control. Fusion 31779

[18] Weiland J 2000 Collective Modes in Inhomogeneous Plasmas, Kinetic and Advanced Fluid Theory (Bristol: Istitute of Physics Publishing)

[19] Tokar M Z et al 2002 Plasma Phys. Control. Fusion 441903

[20] Dominguez R R and Staebler G M 1993 Nucl. Fusion 3351

[21] Waltz R E et al 1995 Phys. Plasmas 22408

[22] Hirshman S P and Sigmar D J 1981 Nucl. Fusion 211079

[23] Shaing K C and Hazeltine R D 1998 Phys. Plasmas 5953

[24] Balescu R 1988 Transport Processes in Plasmas (Amsterdam: North-Holland) vol 2, p 659

[25] Rogister A Private communication

[26] Horton F L Phys. Fluids B 3696

[27] Kalupin D et al 2001 Plasma Phys. Control. Fusion 43945

[28] Kadomtsev B B and Pogutse O P 1971 Nucl. Fusion 1167

[29] Houlberg W A, Shaing K C, Hirshmann S P and Zarnstorff M C 1997 Phys. Plasmas 43230 